\title{
Adolescent attachment, family functioning and depressive symptoms
}

\author{
N Rawatlal, ${ }^{1}$ MA Clin Psych; W Kliewer, ${ }^{1,2} \mathrm{PhD} ; \mathbf{B}$ J Pillay, ${ }^{1} \mathrm{PhD}$ \\ ${ }^{1}$ Department of Behavioural Medicine, University of KwaZulu-Natal, Durban, South Africa \\ ${ }^{2}$ Department of Psychology, Virginia Commonwealth University, Richmond, Virginia, USA
}

Corresponding author: N Rawatlal (rawatlaln@gmail.com)

\begin{abstract}
Background. Adolescence represents a challenging transitional period where changes in biological, emotional, cognitive and social domains can increase the risk of developing internalised problems including subthreshold depression. Adolescent-parent attachment style, perceived support and family functioning may increase risk for depressive symptoms or may reduce such risk. Adolescent-parent attachment, adolescentperceived support from parents and family functioning were examined as correlates of depressive symptom presentation within this age group. Methods. Participants included a maternal parent and an adolescent (65.5\% female) from each family. Adolescents were in Grade 7 ( $n=175)$ or Grade $10(n=31)$. Data were collected through home interviews. The Self-Report of Family Inventory (SFI), Experiences of Close Relationships Scale (ECR), Network of Relationships Inventory (NRI), Children's Depression Inventory (CDI) and Child Behavior Checklist (CBCL) were used to assess depression, parental support and attachment.

Results. Two models were examined: one with adolescent report of depressive symptoms as the outcome and a second with parent report of adolescent internalising symptoms as the outcome. The model predicting adolescent-reported depressive symptoms was significant with older age, higher levels of avoidant attachment, and higher levels of youth-reported dysfunctional family interaction associated with more depressive symptomatology. In the model predicting parent report of adolescent internalising symptoms only higher levels of dysfunctional family interaction, as reported by the parent, were associated with higher levels of internalising symptoms.

Conclusion. Positive family communication, cohesion and support predictive of a secure parent-adolescent attachment relationship reduced the risk of a depressive symptom outcome. Secure adolescents were able to regulate their emotions, knowing that they could seek out secure base attachment relations within their family and from friends during times of stress, buffering against the development of depressive symptoms.
\end{abstract}

S Afr J Psychiatr 2015;21(3):80-85. DOI:10.7196/SAJP.8252

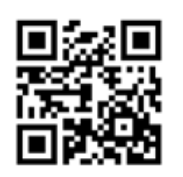

Adolescence is characterised by change in various developmental domains including cognitive, social, affective and biological areas. These simultaneous changes can increase the vulnerability for developing symptoms of depression, known to be prevalent during mid-teens, ${ }^{[1]}$ increasing the risk for negative outcomes, including functional impairment, distress, poorer quality of life, suicidal ideation and the trajectory to a clinical depression in adulthood. ${ }^{[1-3]}$

The World Health Organization identified depression as the most common mental health disorder in adolescence ${ }^{[4]}$ with subthreshold depression increasing the risk for a diagnosis of depression in this age group. ${ }^{[5]}$ Understanding depression and identifying symptoms within this age group is very important in order to render preventive measures and treatment strategies. According to the Diagnostic and Statistical Manual of Mental Disorders, 5th edition (DSM-5), a diagnosis of clinical depression is made when an individual presents with at least five out of nine symptoms of depression (including for example, depressed mood, loss of interest in activities and vegetative shifts) for at least 2 weeks. [6] A subthreshold depression involves functional impairment as a result of a depressive symptom presentation that does not meet the full symptomatic and duration criteria for a diagnosis of depression. ${ }^{[7]}$

In South Africa (SA), Kleintjes et al. ${ }^{[8]}$ reported that, although there were limited data on the prevalence of depression in SA adolescents, their study found that depression was one of the most common disorders reported in childhood and adolescence. Strydom et al. ${ }^{[9]}$ found that even though the prevalence of depression was not high in their sample, the depression rate was still higher in their adolescent sample compared with SA adult rates. Depression is a debilitating diagnosis and a subthreshold depression is known to affect quality of life. ${ }^{[10]}$ For example, some studies show that a diagnosis of depression or a subthreshold depression in adolescence increases the risk for a comorbid psychopathology such as anxiety disorder, bipolar mood disorder, ${ }^{[1]}$ and other functional impairments like poor socialisation skills. ${ }^{[1]}$ In view of the debilitating effects of a subthreshold depression and a major depression diagnosis in adolescence, it is imperative, as a preventive measure, to identify the onset of depressive symptoms early. This study contributes to the literature by examining depressive symptoms in adolescence against the backdrop of family functioning.

\section{Adolescent attachment and depressive symptoms}

Attachment theory emphasises the infant need for proximity-seeking behaviour toward the attachment figure for the primary achievement of protection and security. ${ }^{[12,13]}$ In adolescence, attachment-related behaviour is reconstructed, since the adolescent now becomes less dependent on the parental relationship and more focused on relationships with peers. Although the adolescent still requires the 
attachment figure to provide a secure base, the intensity of this need is modified according to the current developmental needs. ${ }^{[13-15]}$ The adolescent, at this developmental stage, strives for individuation and the attainment of autonomy. Being more cognitively mature, the adolescent can now draw on the patterns of attachment formed in early infant-caregiver interaction, called internal working models, to form interpersonal relationships throughout the lifespan. ${ }^{[13]}$

Attachment patterns generally are classified as secure, insecureavoidant or insecure-anxious types. ${ }^{[16]}$ Secure-type attachment relationships are characterised by optimal attachment during infancy, where the infant felt loved and identified the other as loving, thereby decreasing their vulnerability to negative adjustment. ${ }^{[13]}$ Insecureavoidant and insecure-ambivalent attachment styles are indicative of poor primary caregiver-child interaction, where the reciprocal relationship was characterised as unreliable and inconsistent. Insecure attachments predispose negative psychological and behavioural outcomes in adolescence. ${ }^{[13]}$

Research has consistently established the link, for example, between insecure attachment and depressive symptom presentation in adolescence. ${ }^{[17-19]}$ In a cross-sectional study by Kullick and Petermann, ${ }^{[17]}$ parent and adolescent reports of attachment relationship were investigated in light of depressive symptoms in adolescence. Results showed that insecurely attached adolescents more frequently reported depression. Omidvar et al. ${ }^{[18]}$ investigated the influence of attachment to mother, father and peers as a predictor of depression. They found that in youth, the correlation between parental attachment and depression was higher compared with the correlation between attachment to peers and depression. The authors also found that attachments to both parents and peers significantly predicted depression in adolescence; however, attachment to mother 'predicted higher variances of depression'. Another study highlighting the significant role of maternal attachment on depressive symptoms was carried out by Duchesne and Ratelle. ${ }^{[19]}$ Their longitudinal study compared adolescent reports of attachment security to mothers and fathers in relation to depressive symptom trajectories. They found that secure mother-adolescent attachment, but not father-adolescent attachment, decreased the risk for moderate depressive symptoms in this age group and attachment to both parents decreased the chances of developing more serious depressive symptoms. A further finding showed that consistent parenting by both parents, implicit of a secure attachment, contributed positively to adolescent ability to regulate emotions when coping with stressors. However, another longitudinal study conducted by Gaylord-Harden et al. ${ }^{[20]}$ did not confirm a longitudinal relationship from attachment style to depression, but prospective analysis of the data reported by their adolescent sample revealed a cross-sectional link between attachment and depression. Their study maintained that adolescents who experienced warm maternal relationships were more likely to engage in positive adjustment strategies.

The literature cited shows that the type of attachment relationship (i.e. secure or insecure), as well as to whom the adolescent is attached (mother, father, peers), has a direct influence on a subthreshold symptom outcome in adolescence. To the best of our knowledge, there is limited research into the link between attachment relationships and depressive symptoms in SA adolescents. The literature revealed studies of attachment relationships with other variables and not particularly with depression symptoms. For example, Tomlinson et al. ${ }^{[21]}$ used adult and infant samples to prospectively investigate maternal-infant attachment, using reports from the mother, and infant attachment as assessed by using Ainsworth's strange situation experiment. One of their findings was that even against the backdrop of disadvantaged socioeconomic status, mother-infant attachment could be secure. Another study by Chetwin ${ }^{[22]}$ used a qualitative methodology to investigate the impact of the apartheid system on quality of attachment relationships reported by adult South Africans. Overall findings from this study showed that the quality and stability of early childhood relationships are related to the development of secure attachment and successful autonomy in adulthood.

The current study contributes to the literature by examining associations between two types of attachment relationships and depressive symptoms in a cohort of SA adolescents in a low-income setting.

\section{Family functioning, adolescent attachment and adolescent depressive symptoms}

The quality of family functioning in terms of communication, cohesion and support has an influence on attachment relationships. The influence between these family processes and adolescentparent attachment can influence a depressive symptom outcome. For example, Kapanee and $\mathrm{Rao}^{[23]}$ examined the effect of attachment styles in relation to family functioning and depressive symptoms. They found that participants who reported greater levels of family cohesion and better communication patterns within their families also reported greater levels of secure attachment and were at a lower risk for the development of depressive symptoms, because secure attachment serves as a protective factor against negative psychological outcomes, such as symptoms of depression. Therefore, insecure adolescent-parent attachments, evident of low family cohesion and communication, presumably increase the risk for the development of adolescent subthreshold depression. In contrast, Shaw and Dallos ${ }^{[24]}$ point out that insecure attachment style and depressive symptoms in adolescence cannot simply be explained as having a cause-andeffect relationship. Instead they maintain that an insecure attachment pattern can increase adolescent susceptibility to emotional distress, since the individual may become more vulnerable in stressful conditions such as family conflict. Attachment relationship and family functioning, then, can have a bidirectional relationship in influencing the presentation of depressive symptoms in adolescence.

In other studies Fonagy et al. ${ }^{[25]}$ found that with a caregiver's appropriate mirroring, children gain awareness of their own internal states and also of internal states of others. The caregiver's capacity to monitor and reflect the child's moment-to-moment states (maternal reflective function) is a strong predictor of both secure attachment and adaptive, coherent mental organisation. ${ }^{[25,26]}$ Thus secure attachment is the outcome of successful containment and leads to better selfregulation of behaviour. ${ }^{[27]}$

Studies confirm that the quality of family functioning is associated with a depressive symptom presentation in adolescence. For example, Briere et al. ${ }^{[28]}$ conducted a longitudinal investigation into the association between family communication and conflict on depressive symptoms reported by adolescents. They found that adolescents perceived that conflict with parents and depressive symptoms predicted each other, and in turn depressive symptoms predicted poorer parent-adolescent communication. However, better communication did not predispose 
a decrease in depressive symptoms. Piko et al. ${ }^{[29]}$ reported that higher levels of adolescent-perceived parental support significantly correlated with lower levels of depressive symptoms. Similarly, a longitudinal study conducted by Sze et al..$^{[30]}$ showed that close and caring families, reflective of high levels of family cohesion, communication and support, are associated with lower levels of depressive symptomatology in adolescence. Queen et al. ${ }^{[31]}$ investigated mothers' and fathers' reports of family cohesion on depressive symptoms reported by adolescents. Their findings indicated a strong correlation between family cohesion and depressive symptoms in their adolescent sample. Delay et al. ${ }^{[32]}$ examined adolescent reports of the link between parental support, family conflict and depressive symptoms. Their study showed that, ultimately, parental support buffered against the development of depressive symptoms in adolescence. They found that parental support showed a more significant correlation with depressive symptoms than family conflict, whereby a lack of support from parents was associated with depression, even where the level of family conflict was low.

The current study contributes to the literature by examining associations between attachment patterns, as reported by adolescents, perceived adolescent reports of parental support, and adolescent and parent reports of family functioning, with adolescent-reported depressive symptoms and parent-reported internalising symptoms within the SA context.

\section{Methods}

This study is part of a larger collaborative study (Project CARE: Community Assessment of Risk and Resilience) between the University of KwaZulu-Natal (UKZN) and Virginia Commonwealth University (VCU) in Richmond, VA, USA, investigating associations between cumulative risk and adolescents' behavioural, psychological, cognitive and physiological functioning.

\section{Participants}

Participants were recruited from public schools in Durban, KwaZuluNatal. Communities included were: Umlazi, KwaMashu, Chatsworth, Phoenix, Umbilo, Newlands and Wentworth. Families $(N=206)$ interviewed during 2012 - 2013 were included in the sample. Participants were adolescents, either in Grade 7 (primary school) $(n=175)$ or Grade 10 (secondary school) $(n=31)$, together with their maternal parent or primary caregiver. Adolescents ranged in age from 9 to 18 years (mean (standard deviation $(\mathrm{SD})=13.02(1.42)$ years) and $65.6 \%$ were female. Caregivers ranged in age from 21 to 75 (mean $(\mathrm{SD})=41.64$ (9.94) years). Most $(72.3 \%)$ caregivers were the adolescent's biological mother; grandmothers (12.6\%), aunts $(6.8 \%)$, stepmothers $(2.9 \%)$, sisters (1.5\%), adopted mothers $(1.0 \%)$, and cousins $(0.5 \%)$ also participated.

\section{Procedure}

Participant recruitment was done via school visits. Students were addressed either in a special assembly or in their individual classrooms where they were briefed about the study from a standardised script. They were given information packs consisting of a letter addressed to their parents describing the study, and assent and consent forms. Interviews were conducted at participants' homes using caregiver and youth interviews.The caregiver interview covered topics including demographics, their reports of their child's internalising depressive symptomatology, and family cohesion and communication (family functioning). The youth interview covered topics such as: adolescent attachment, family functioning, perceived support from caregivers, cognitive, behavioural and affective symptoms of depression and demographic information such as age and grade. At the conclusion of the home interview, caregivers received ZAR150 for their time in the form of a shopping voucher. At the conclusion of the home interview, youth received ZAR50 in the form of a shopping voucher. Based on SA standards and norms, this is an appropriate level of compensation. Ethical approval for this study was obtained from the Biomedical Research Ethics Committee at UKZN and the KwaZulu-Natal provincial Department of Education, as well as the Ethics Review Board at VCU. Thereafter, consent was requested and obtained from the relevant school authorities, teachers and parents. Participation was voluntary.

\section{Measures}

\section{Self-report of Family Inventory (SFI)}

The SFI ${ }^{[33]}$ measures family cohesion, communication and conflict. It is a 36-item self-report questionnaire (by caregiver and youth) which has been used to measure family functioning in different populations and cultures. ${ }^{[33]}$ Maternal caregivers answered the same questions as the youth did with minor changes in the wording where necessary. The alpha coefficients for the scale ranged from 0.84 to 0.88 , indicating adequate internal consistency. ${ }^{[34]}$ Test-retest reliability coefficients for 30 - 90 days ranged from 0.84 to 0.87 for family health/ competence, 0.50 to 0.59 for conflict, 0.50 to 0.70 for cohesion, 0.79 to 0.89 for expressiveness, and 0.41 to 0.49 for directive leadership. The SFI has continually demonstrated good validity as shown in correlations with various other scales measuring similar conceptual domains. For example, good validity was reported with correlations of 0.62 or better for the competence scale scores between the SFI and the Beavers Interactional Competence scale. ${ }^{[35]}$ Cronbach's alphas in the current study were 0.88 for caregiver reports and 0.89 for youth reports.

\section{Experiences of Close Relationships Scale (ECR)}

The $\mathrm{ECR}^{[13]}$ (completed by youth) measures attachment style and close relationships. It contains 36 items, measuring attachment styles of respondents using two bidirectional subscales namely avoidance of intimacy and closeness and anxiety about being rejected, feelings of jealousy and fears of abandonment. The avoidance and anxiety factors in combination contribute to data about four attachment styles which are secure, preoccupied, dismissing, and fearful attachment. In the present study, the continuous anxious and avoidant subscales were used. The internal consistencies reported in prior studies were good: anxiety: $\alpha=0.90$ and avoidance: $\alpha=0.91$, confirming the reliability of the ECR. ${ }^{[36,37]}$ Conradi et al.$^{[37]}$ compared the psychometric properties of the Dutch ECR and the original American ECR and found that the internal and external validity was adequate, which is suggestive of broader applicability. Cronbach's alphas in the current study were 0.84 for anxious attachment and 0.82 for avoidant attachment.

\section{Network of Relationships Inventory (NRI)}

The NRI ${ }^{[38]}$ (reported by youth) is conceptualised within Robert Weis's theory on social provisions. It describes six basic relationship qualities, namely: (i) attachment which involves affection, security and intimate disclosure; (ii) reliable alliance, which implies a trustworthy 
and dependable bond; (iii) enhancement of worth, where one feels validated and one's competence is affirmed; (iv) social integration - interpersonal sharing and companionship; (v) guidance, which relates to support and advice; and (vi) opportunity for nurturance - caring for one another. For the current study, the quality of relationship between the youth and the primary maternal caregiver, and the youth and the father figure was assessed using seven questions from the original NRI. Youth use the same set of characteristics to assess their relationship with the primary maternal caregiver, the father figure and the significant other adult in their lives. Furman and Buhrmester ${ }^{[38]}$ found that the internal consistency of the scales was satisfactory (Cronbach's alpha $=0.80)$. Plybon and Kliewer $^{[39]}$ also reported satisfactory internal consistency (Cronbach's alpha $=0.77$ ) using the same seven-item measure used in the present study, as well as evidence of validity. Cronbach's alphas in the current study were 0.89 for support from mother and 0.92 for support from father.

\section{Children's Depression Inventory (CDI)}

The $\mathrm{CDI}^{[40]}$ comprises 27 items measuring factors such as: negative moods (e.g. feelings of sadness, feelings of tearfulness, worrying about bad things); social avoidance and isolation; anhedonia; low self-esteem; and self-dislike. ${ }^{[40]}$ The CDI has been found to have a high internal consistency with alpha values and split half correlations above 0.8. Van Beek et al. ${ }^{[41]}$ reported good reliability (Cronbach's alpha $=0.81$ ). Retest after 1 month demonstrated significant correlation, $r=0.67, p=0.001$. Kovacs $^{[40]}$ reported high reliability and internal consistency with coefficients ranging from 0.71 to 0.89 . The CDI demonstrated significant validity when correlated with the Standardized Psychiatric Interview. ${ }^{[42]}$ Cronbach's alpha in the current study was 0.86 .

\section{Child Behaviour Checklist (CBCL)}

The 14-item depression-anxiety subscale of the $\mathrm{CBCL},{ }^{[43]}$ completed by the parent, was used to assess internalising symptoms. The CBCL demonstrates adequate reliability on the anxious/depression scale $(0.86-0.88) \cdot{ }^{[43]}$ According to Nakamura et al., ${ }^{[44]}$ good internal consistency and convergent and divergent validity of the $\mathrm{CBCL}$ were found when compared with other scales, e.g. the CDI. Cronbach's alpha for the anxiety/depression subscale was 0.81 in the current study.

\section{Results}

Table 1 presents descriptive information on and correlations among the study variables. Owing to high correlations among the predictor variables, regression analyses were used to examine the unique contributions of the predictor variables of anxious and avoidant attachment, perceived support from mother and father, and caregiver and adolescent reports of family functioning on depressive symptoms in adolescence. Mean substitution was used for missing data in order to increase power. Two models were examined: one with adolescent report of depressive symptoms as the outcome and a second with parent report of adolescent internalising symptoms as the outcome.

For the regression predicting adolescent report of depressive symptoms, $F_{8,163}=11.57$, $p<0.001$, therewerethreesignificant predictors in the model: age $(\beta=0.14, p<0.05)$, avoidant attachment, $(\beta=0.34, p<0.001)$, and youth report of family dynamics $(\beta=-0.20, p<0.05)$. As indicated by the beta weights, older age, higher levels of avoidant attachment, and higher levels of youth-reported dysfunctional family interaction were associated with more depressive symptomatology as rated by youth. For the regression predicting caregiver report of adolescent internalising symptoms, $F_{8,161}=2.00, \quad p<0.05$, caregiver report of family dynamics $(\beta=-0.27, p<0.001)$ was the only significant predictor. Higher levels of caregiver-reported dysfunctional family interaction was associated with more caregiver-rated internalising symptoms.

\section{Discussion}

Our research examined adolescent attachment, adolescent-perceived support from parents and reports of family functioning from the adolescent and primary caregiver as correlates of a subthreshold depressive presentation.

As hypothesised, and consistent with other studies, ${ }^{[17,45,46]}$ our findings reveal that depressive symptoms in adolescence are significantly correlated with an insecure adolescent-parent attachment. While secure attachment in adolescence is related to positive outcomes including positive coping skills and emotion regulation, ${ }^{[4]}$ several theories exist that explain the robust association between depression and insecure attachment style. Allen and Land ${ }^{[14]}$ explain that adolescence is characterised by transformations in the emotional and behavioural structures within the milieu of advancements in the cognitive domain. As a result progressive logical and abstract reasoning abilities promote critical evaluation of the self and significant other attachment relationship, which increases the risk for depressive symptom outcomes. The theory further explains that an insecure parent-infant attachment results in the development of insecure internal working models. An insecure working model, developed during the infant-caregiver relationship, predisposes inabilities in managing future complex attachment relations in adolescence, since the adolescent draws on this internalised schema in peer interaction. This process can be a precursor to a depressive symptom experience by the adolescent. Implicit in Allen and

Table 1. Descriptive information on and correlations among study variables

\begin{tabular}{|c|c|c|c|c|c|c|c|c|}
\hline & 1 & 2 & 3 & 4 & 5 & 6 & 7 & 8 \\
\hline 1. ECRanx & & $0.23^{*}$ & -0.13 & -0.04 & $-0.17^{\dagger}$ & $-0.16^{\dagger}$ & $0.17^{\dagger}$ & 0.01 \\
\hline 2. ECRavd & & & $-0.45^{*}$ & $-0.37^{*}$ & 0 & $-0.57^{*}$ & $0.52^{*}$ & 0.07 \\
\hline 3. NRIsupm & & & & $0.50^{*}$ & -0.04 & $0.45^{*}$ & $-0.39^{*}$ & -0.06 \\
\hline 4. NRIsupf & & & & & 0.02 & $0.43^{*}$ & $-0.23^{\ddagger}$ & -0.06 \\
\hline 5. SFIc & & & & & & 0.06 & 0.02 & -0.23 \\
\hline 6. SFIy & & & & & & & $-0.45^{\star}$ & -0.12 \\
\hline 7. CDI & & & & & & & & $0.20^{\ddagger}$ \\
\hline \multicolumn{9}{|l|}{ 8. CBCL } \\
\hline Mean & 66.99 & 52.79 & 27.96 & 25.43 & 72.88 & 70.29 & 9.13 & 5.01 \\
\hline SD & 21.89 & 21.00 & 6.63 & 8.02 & 13.17 & 7.55 & 7.06 & 4.63 \\
\hline \multicolumn{9}{|c|}{$\begin{array}{l}E C R a n x=\text { anxious attachment; } \mathrm{ECRavd}=\text { avoidant attachment; NRIsupm }=\text { perceived support from mo } \\
\text { port from father; SFIc }=\text { family functioning, reported by caregiver; } \mathrm{SFIy}=\text { family functioning, reported } \\
\text { Depressive Inventory; } \mathrm{CBCL}=\text { anxious-depressive symptom subscale of the Child Behavior Checklist. } \\
{ }^{*} p<0.001 \\
{ }^{+} p<0.05 \\
{ }^{p} p<0.01\end{array}$} \\
\hline
\end{tabular}


Land's theory ${ }^{[14]}$ is the influence of neurological changes in the transition from childhood into adolescence of a depressive symptom presentation. The current research did not investigate cognitive perspectives of adolescent development in the understanding of attachment style and depressive symptoms. Future research might focus on neuropsychological correlates of attachment and depression to explain the relationship between attachment style and depressive symptoms as outlined in Allen and Land's theory.

In another model highlighting the relationship between attachment and depressive symptoms, Ronnlund and Karlsson ${ }^{[45]}$ explain that insecurely attached infants will be preoccupied with attachment experiences as they grow, resulting in feelings of relationship distress as adolescents. This preoccupation with interpersonal interactions will make the adolescent continually seek out peer relationships, causing ongoing distress, making them more vulnerable toward the development of symptoms of depression. Finding support for the above-mentioned model, Ronnlund and Karlsson ${ }^{[45]}$ examined dimensions of attachment and depressive symptom problems as reported by their adolescent sample. They found that adolescents who are preoccupied with interpersonal relationships and feel a general uneasiness with relationships, characteristic of insecure attachments, were more likely to report depressed mood. Duchesne and Ratelle ${ }^{[19]}$ also found support for the above-mentioned model in their research. They reported that the secure infant-caregiver attachment acts as a protective factor against the development of depressive symptomatology in adolescence.

Applied within an SA context, the models highlighted above perhaps lend explanation to the supported correlation in our study. Limited SA research on adolescent attachment style and depressive symptoms was sourced. It is recommended that future research into the relationship between adolescent attachment style and subthreshold depression be considered.

In our study family dynamics were significantly correlated with depressive symptoms, concurring with findings from Queen et al. ${ }^{[31]}$ The family dynamics of cohesion, support and communication appear to coincide with the adolescent-parent attachment dynamic. More of these dynamics within the family is related to a greater degree of attachment. Less communication, cohesion and support within the family contributes to less attachment, thereby increasing the adolescent's vulnerability to depressed mood symptoms. ${ }^{[17,45,47]}$ In contrast, Roubinov and Luecken ${ }^{[48]}$ found that family conflict, in particular, was a significant predictor of depressive symptoms. Briere et $a l .{ }^{[28]}$ reported that adolescent depressive symptoms predicted poorer adolescent-parent communication but improved communication did not decrease depressive symptoms, while parental support acted as a buffer against depressive symptoms in another study. ${ }^{[32]}$

Limitations of the study included the cross-sectional design, which did not allow us to examine trajectories or changes in depressive symptoms over time. Future studies using longitudinal designs may be helpful in determining whether variations in family functioning and changes in attachment patterns are associated with changes in depressive symptoms across adolescence. Although both adolescents and their parents reported on depressive symptoms, these reports were not highly correlated. This pattern is often observed in the literature, ${ }^{[49]}$ and may reflect the fact that adolescents have greater awareness of their internal states than adults around them, such as parents or teachers. Furthermore, only adolescent reports of family functioning were associated with adolescent-reported depressive symptoms; only parent reports of family functioning were associated with parent-reported internalising symptoms. This pattern of finding suggests that at least some of the association between attachment, support from parents, family functioning and adolescent depressive symptoms is due to shared method variance. Multimethod studies that include multiple sources of information as well as multiple methods of assessing constructs may help to tease apart true findings from those that are artefacts of methodology. Nonetheless, this study contributes to the literature on SA youth in a low-income setting by documenting associations between insecure attachment, family functioning and depressive symptoms in youth.

Funding. This study was funded by a Global Education Grant from Virginia Commonwealth University, USA, and from the Department of Behavioural Medicine at the University of KwaZulu-Natal, Durban, South Africa. The views expressed in the article are those of the authors and not an official position of the institution or funder.

\section{References}

1. Wesselhoeft R, Sorensen MJ, Heiervang ER, Bilenberg N. Subthreshold depression in children and adolescents - a systematic review. J Affect Disorders 2013;151:7-22. [http://dx.doi. org/10.1016/j.jad.2013.06.010]

2. Bertha A, Balazs J. Subthreshold depression in adolescence: A systematic review. Eur Child Adolesc Psychiatry 2013;22:589-603. [http://dx.doi.org/10.1007/s00787-013-0411-0]

3. Schwartz OS, Dudgeon P, Sheeber LB, et al. Parental behaviours during family interactions predict changes in depression and anxiety symptoms during adolescence. J Abnorm Child Psychol 2012;40:59-71. [http://dx.doi.org/10.1007/s10802-011-9542-2]

4. World Health Organization. Adolescent Mental Health. Mapping actions of nongovernmental organizations and other international development organizations. Geneva: World Health Organization, 2012. http://whqlibdoc.who.int/publications/2012/9789241503648_eng.pdf (accessed 7 July 2014).

5. Thapar A, Collishaw S, Pine DS, Thapar AK. Depression in adolescence. Lancet 2012;379:1056-1067. [http://dx.doi.org/10.1016/S0140-6737(11)60871-4]

6. American Psychiatric Association. Diagnostic and Statistical Manual of Mental Disorders, 5th ed. (DSM-5). Washington, DC: American Psychiatric Association, 2013:155-188.

7. Gotlib IH, Lewinsohn PM, Seeley JR. Symptoms versus a diagnosis of depression: Differences in psychosocial functioning. J Consult Clin Psychol 1995;63:90-100.

8. Kleintjes S, Flisher AJ, Railoun A, Lund C, Molteno C, Robertson BA. The prevalence of mental disorders among children, adolescents and adults in the Western Cape, South Africa. S Afr Psychiatry Rev 2006;9:157-160.

9. Strydom MAA, Pretorius PJ, Joubert G. Depression and anxiety among Grade 11 and Grade 12 learners attending schools in central Bloemfontien. S Afr J Psychiatr 2012;18:84-88. [http:// dx.doi.org/10.7196/SAJP.356]

10. Karsten J, Penninx BWJH, Verboom CE, Nolen WA, Hartman CA. Course and risk factors of functional impairment in subthreshold depression and anxiety. Depression Anxiety 2012;30:386-394. [http://dx.doi.org/10.1002/da.22021]

11. Naiker K, Galambos NL, Zeng Y, Senthilselvan A, Colman I. Social, demographic, and health outcomes in the 10 years following adolescent depression. J Adolesc Health 2013;52:533-538. [http://dx.doi.org/10.1016/j.jadolhealth.2012.12.016]

12. Bowlby J. A Secure Base: Parent-child Attachment and Healthy Human Development. New York: Basic Book, 1988: 9-117.

13. Mikulincer M, Shaver PR. Attachment in Adulthood: Structure, Dynamics and Change. New York, London: Guildford Press, 2007: 3-209.

14. Allen JP, Land D. Attachment in adolescence. In: Cassidy J, Shaver P, eds. Handbook of Attachment: Theory, Research and Clinical Implications. New York: Guildford Press, 1999:319-335.

15. Dubois-Comtois K, Cyr C, Pascuzzo K, Lessard M, Poulin C. Attachment theory in clinical work with adolescents. J Child Adolesc Behav 2013;3:1-8. [http://dx.doi.org/10.4172/jcalb.1000111]

16. Ainsworth M. Patterns of infant-mother attachments: Antecedents and effects on development. Bull N Y Acad Med 1985;69:771-779.

17. Kullik A, Petermann F. Attachment to parents and peers as a risk factor for adolescent depressive disorders: The mediating role of emotion regulation. Child Psychiatry Hum Dev 2013;44:537-548. [http://dx.doi.org/10.1007/s10578-012-0347-5]

18. Omidvar B, Bahrami F, Fatehizade M, et al. Attachment quality and depression in Iranian adolescents. Psychol Stud 2012;309-315. [http://dx.doi.org/10.1007/7s12646-014-0250-1]

19. Duchesne S, Ratelle CF. Attachment security to mothers and fathers and the developmental trajectories of depressive symptoms in adolescence: Which parent for which trajectory? J Youth Adolesc 2014;43:614-654. [http://dx.doi.org/10.1007/s10964-013-0029-z]

20. Gaylord-Harden NK, Taylor JJ, Campbell CL, et al. Maternal attachment and depressive symptoms in urban adolescents: The influence of coping strategies and gender. J Child Adolesc Psychol 2009;38(5):685-695. [http://dx.doi.org/10.1080/15374410903103569]

21. Tomlinson M, Cooper P, Murray L. The mother-infant relationship and infant attachment in a South African peri-urban settlement. Child Dev 2005;76:1044-1054. 


\section{ners

22. Chetwin F. Attachment for a group of black South African children during apartheid. South Africa, Gauteng, 2010. http://hdl.handle.net/10539/11252 (accessed 11 August 2014).

23. Kapanee ARM, Rao K. Attachment style in relation to family functioning and distress in college students. J Indian Acad Appl Psychol 2007;33:15-21.

24. Shaw SK, Dallos R. Attachment and adolescent depression: The impact of early attachment experiences. Attach Hum Dev 2005;7:409-424.

25. Fonagy P, Steele H, Moran G, Steele M, Higgitt A. The capacity for understanding mental states: The reflective self in parent and child and its significance for security of attachment. Infant Ment Health J 1991;13:200-217.

26. Fonagy P, Steele M, Steele H, Higgitt A, Target M. The Emmanuel Miller Memorial Lecture 1992. The theory and practice of resilience. J Child Psychol Psychiatry 1994;35:231-257.

27. Fonagy P, Target M. Attachment and reflective function. Dev Psychopathol 1997;9:679-700.

28. Briere FN, Archambault K, Janosz M. Reciprocal prospective associations between depressive symptom and perceived relationship with parents in early adolescence. Can J Psychiatry 2013;58(3):169-176.

29. Piko BF, Luszczynska A, Fitzpatrick K. Social inequalities in adolescent depression: The role of parental social support and optimism. Int J Soc Psychiatry 2012;59:474-481. [http://dx.doi. org/10.101177/0020764012440788

30. Sze TM, Hsieh PJ, Lin SH, Chen IJ. Sex differences in the development of perceived family cohesion and depressive symptoms in Taiwanese adolescents. Psychol Rep: Mental and Physical Health 2013;113(1): 54-72. [http://dx.doi.org/10.2466/10.02PR0.113x18z2]

31. Queen AH, Steward LM, Ehrenreich-May J, Pincus DB. Mothers' and fathers ratings of family relationship quality: Associations with preadolescent and adolescent anxiety and depressive symptoms in a clinical sample. Child Psychiatry Hum Dev 2013;44:351-360. [http://dx.doi org/10.1007/s10578-012-0329-7]

32. Delay D, Hafen CA, Cunha JM, et al. Perceptions of parental support buffer against depression for Brazilian youth with interpersonal difficulties. Int J Behav Dev 2012;37(1):29-34. [http:// dx.doi.org/10.1177/0165025412454031]

33. Hamson RB, Hulgus YF, Beavers WR. Comparisons of self-report measures of the Beavers Systems Model and Olson's Circumplex Model. J Fam Psychol 1991;4:326-240.

34. Beavers R, Hampson RB, Hulgus YF. Beavers Systems Manual: 1990 Edition. Dallas, TX: Southwest Family Institute, 1990

35. Beavers R, Hampson RB. The Beavers systems model of family therapy. J Fam Ther 2000;22:128-143.

36. Cohen E, Solomon Z, Zerach G. The implication of combat induced stress reaction, PTSD, and attachment in parenting among war veterans. J Fam Psychol 2011;25:688-698.
37. Conradi HJ, Gerslma C, van Duijn M, de Jonge P. Internal and external validity of the Experience in Close Relationships questionnaire in an American and two Dutch samples. Eur Psychiatry 2006;20:258-269.

38. Furman W, Buhrmester D. Children's perceptions of the personal relationships in their social network. Dev Psychol 1985;6:1016-1024.

39. Plybon LE, Kliewer W. Neighborhood types and externalizing behavior in urban school-age children: Tests of direct, mediated, and moderated effects. J Child Fam Stud 2001;10:419-437.

40. Kovacs M. The Children's Depression Inventory (CDI) manual. North Tonawanda, NY: MultiHealth Systems, 1992

41. Van Beek Y, Hessen DJ, Hutteman R, Verhulp EE, van Leuven M. Age and gender differences in depression across adolescence: Real or 'bias'? J Child Psychol Psychiatry 2012;53:973-985. [http://dx.doi.org/10.1111/j.1469-7610.2012.02553.x].

42. Fundudis T, Berney TP, Kolvin I, et al. Reliability and validity of two self-rating scales in the assessment of childhood depression. Br J Psychiatry 1991;159:36-40.

43. Achenbach TM. Integrative guide for the 1991 CBCL 4-18, YSR, and TRF profiles. Burlington, VT: University of Vermont, Department of Psychiatry 1991, 1-6.

44. Nakamura BJ, Ebesutani C, Bernstein A, Chorpita BF. A psychometric analysis of the child behavior checklist DSM-oriented scales. J Psychopathol Behav Assess 2009;31:178-189. [http://dx.doi.org/10.1007/s10862-008-9119-8].

45. Ronnlund M, Karlsson E. The relation between dimensions of attachment and internalizing or externalizing problems during adolescence. J Genet Psychol 2006;167:47-63. [http://dx.doi. org/10.3200/GNTP.167.1.47-63].

46. Brenning KM, Soenens B, Braet C, Bosmans G. Attachment and depressive symptoms in middle childhood and early adolescence: Testing the validity of the emotion regulation model of attachment. Pers Relat 2012;19:445-464. [http://dx.doi.org/10.1111/j.14756811.2011.01372.x].

47. Miller PM, Commons ML. The benefits of attachment parenting for infants and children: A behavioural developmental view. Behav Dev Bull 2010;10:1-14

48. Roubinov DS, Luecken LJ. Family conflict in childhood and adolescence and depressive symptoms in emerging adulthood: Mediation by disengagement coping. J Divorce Remarriage 2013;54(7):576-595. [http://dx.doi.org/10.1080/10502556.2013.828988].

49. De Los Reye A, Kazdin AE. Informant discrepancies in the assessment of childhood psychopathology: A critical review, theoretical framework, and recommendation for further study. Psychol Bull 2005:4:483-509. [http://dx doi.org/10.1037/00332009.131.4.483

\section{The Oxford Handbook of PSYCHIATRIC ETHICS}

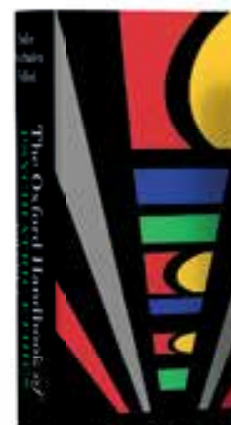

The Oxford $\mathrm{f}$

PSYCHL

ETHIC

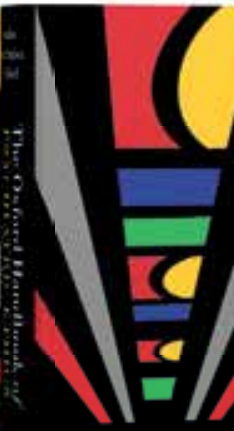

The Oxford Handbook of

PSYCHIATRIC ETHICS

votent 1
Edited by Prof John Z. Sadler,

Prof Werdie (C.W.) Van Staden, and Prof K.W.M. (Bill) Fulford

Psychiatric ethics is an excellent framework in which to examine social changes in the field of psychiatry over the past 25 years, changes which are dramatic in nature and profound in impact. The Oxford Handbook of Psychiatric Ethics is the most comprehensive treatment of the field in history, with global coverage of this important field.

9780199663880 | Hardback|£175.00 |July 2015| 2000 pp |2 volumes

www.oup.com/uk/medicine 